\title{
Intracellular Drug Bioavailability: Effect of Neutral Lipids and Phospholipids
}

Andrea Treyer, ${ }^{\dagger}$ André Mateus, ${ }^{\dagger}$ Jacek R Wiśniewski, ${ }^{\ddagger \oplus}$ Hinnerk Boriss, ${ }^{\S}$ Pär Matsson, ${ }^{\dagger}$ and Per Artursson $*, \dagger, \|, \perp$

\author{
${ }^{\dagger}$ Department of Pharmacy, Uppsala University, Uppsala 75123, Sweden \\ ${ }^{\ddagger}$ Biochemical Proteomics Group, Department of Proteomics and Signal Transduction, Max Planck Institute of Biochemistry, \\ Martinsried 82152, Germany \\ ${ }^{\S}$ Sovicell GmbH, Leipzig 04103, Germany \\ "Science for Life Laboratory Drug Discovery and Development Platform (SciLifelab DDD-P), Uppsala 75123, Sweden \\ ${ }^{\perp}$ Uppsala University Drug Optimization and Pharmaceutical Profiling Platform (UDOPP), Uppsala University, Uppsala 75123, Sweden
}

\section{Supporting Information}

\begin{abstract}
Intracellular unbound drug concentrations are the pharmacologically relevant concentrations for targets inside cells. Intracellular drug concentrations are determined by multiple processes, including the extent of drug binding to intracellular structures. The aim of this study was to evaluate the effect of neutral lipid (NL) and phospholipid (PL) levels on intracellular drug disposition. The NL and/or PL content of 3T3-L1 cells were enhanced, resulting in phenotypes (in terms of morphology and proteome) reminiscent of adipocytes (high

1. Cell characterization

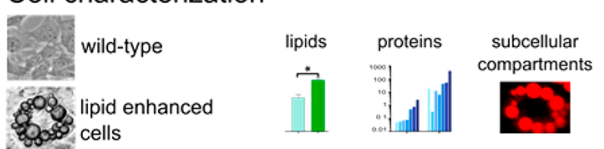

2. Intracellular drug bioavailability$$
F_{i c}=f_{u, c e l l} \cdot K p
$$

$\Rightarrow$ Phospholipid content is a major determinant of $f_{u, c e l l}$ $\mathrm{NL}$ and PL) or mild phospholipidosis (only high PL). Intracellular bioavailability $\left(F_{\mathrm{ic}}\right)$ was then determined for 23 drugs in these cellular models and in untreated wild-type cells. A higher PL content led to higher intracellular drug binding and a lower $F_{i c}$. The induction of NL did not further increase drug binding but led to altered $F_{\text {ic }}$ due to increased lysosomal $\mathrm{pH}$. Further, there was a good correlation between binding to beads coated with pure PL and intracellular drug binding. In conclusion, our results suggest that PL content is a major determinant of drug binding in cells and that PL beads may constitute a simple alternative to estimating this parameter. Further, the presence of massive amounts of intracellular NLs did not influence drug binding significantly.
\end{abstract}

KEYWORDS: intracellular drug bioavailability, lipid, phospholipid, drug binding, membrane partitioning, proteomics, 3T3-L1, unbound concentration

\section{INTRODUCTION}

Intracellular unbound drug concentrations are the pharmacologically relevant concentrations for targets in the cell interior. ${ }^{1}$ The intracellular concentrations are not necessarily equal to the unbound concentrations in the blood, and they are affected by active transport and clearance processes within the cell. Furthermore, drug exposure may vary depending on the subcellular localization of the target, for example, as a result of concentration gradients across organelle membranes. Quantification of the intracellular unbound drug exposure is therefore of great importance in drug discovery and development to improve prediction of drug efficacy and toxicity.

We and others have recently developed methods to quantify intracellular unbound drug concentrations. ${ }^{2-10}$ Our small-scale approach can be applied in drug discovery settings and involves parallel measurements of total intracellular drug concentration $\left(C_{\text {cell }}\right)$ and the fraction of unbound drug in the cell $\left(f_{\mathrm{u}, \mathrm{cell}}\right){ }^{2}$ These two parameters are then combined to obtain an estimate of intracellular bioavailability $\left(F_{\text {ic }}\right)$, i.e., the ratio between the intracellular unbound concentration, which is available to elicit pharmacological effects inside the cell, and the extracellularly applied concentration. When $F_{\text {ic }}$ is equal to 1 , the unbound concentration of a drug in the cell interior is equal to the concentration applied to the cell, whereas lower or higher values indicate, respectively, restricted or increased intracellular bioavailability of the drug. $F_{\text {ic }}$ has been used to study the effect of drug transporters on intracellular drug concentrations $s^{3,6,7}$ and to study the importance of intracellular drug concentration for target engagement. ${ }^{11}$

Numerous factors can affect $F_{\text {ic }}$. These include drug transporters and drug-metabolizing enzymes, ${ }^{3,5-7}$ sequestration of the drug into organelles (usually endolysosomes or mitochondria), ${ }^{8,12-14}$ and the degree of binding to cellular components, such as lipids and proteins. This binding occurs mostly nonspecifically to the cell membranes involved in lipid

Received: January 19, 2018

Revised: April 24, 2018

Accepted: April 30, 2018

Published: April 30, 2018 
partitioning. ${ }^{15-17}$ Studies of drug interactions with phospholipid membranes ${ }^{18,19}$ and tissue lipids ${ }^{20,21}$ indicate the importance of phosphatidylcholine and phosphatidylserine in drug distribution. However, the individual influence of major lipid classes on $f_{\mathrm{u} \text {,cell }}$ has not been fully investigated. Here, we therefore used our small-scale methodology to investigate the effect of phospholipid (PL) and neutral lipid (NL) concentrations on $F_{\mathrm{ic}}$. The studies were performed in 3T3-L1 fibroblasts. This is a cell line that can be transformed into adipocytes containing NL droplets $^{22}$ and that, as shown here, can also be transformed to accumulate PLs. Our results indicate concentrations of PLs, but not those of NLs, to be a major determinant of the unbound intracellular drug fraction. We also show that $f_{\text {u,cell }}$ can be predicted from the drug affinities for pure phosphatidylcholine.

\section{EXPERIMENTAL SECTION}

Chemicals. Compounds were obtained from Sigma-Aldrich, Fluka, Tocris, ICN Biomedicals, and from the Prestwick library (Prestwick Chemical) at their highest available degree of purity ( $\geq 95 \%$ ). DMSO stocks (10 mM, or highest possible solubility if lower) were kept at $-20{ }^{\circ} \mathrm{C}$ after preparation and were used for a maximum of four freeze-thaw cycles.

Compound Selection. We aimed to study $F_{\text {ic }}$ using a representative compound set for the chemical space of small-molecule drugs. We first calculated 334 ADME-related molecular properties for the complete Prestwick library $(n=1197)$ using ADMET predictor (Simulations Plus, version 7.2). These properties were used as features in a principal component analysis in Simca-P (Umetrics, version 12.0). A preliminary subset of 51 compounds, spread over the chemical space of the full library, was selected for a first round of experimental screening (Figure S1). After the compounds for which UPLC-MS/MS detection was problematic (due to poor compound ionization or chromatographic retention) or for which quality requirements in terms of recovery or stability were problematic were excluded, the set consisted of 23 representative compounds. The calculated octanolbuffer coefficient at $\mathrm{pH} 7.4\left(\log \mathrm{D}_{7.4}\right)$ ranged from -0.7 to 5.0, polar surface area (PSA) from 28.2 to $146.0 \AA^{2}$, and molecular weight (MW) from 194.2 to 628.8. Six of the compounds were anionic, eight were cationic, and nine were neutral at $\mathrm{pH} 7.4$.

Cell Culture. 3T3-L1 cells (ATCC) were grown in culture medium (Dulbecco's Modified Eagle's Medium, DMEM) containing $4.5 \mathrm{~g} / \mathrm{L}$ glucose (Thermo Fisher, no. 41966) and supplemented with $10 \%$ bovine calf serum (BCS) (ATCC, no. $30-2030)$ ). Cells were passaged at $80 \%$ confluency at a ratio of 1:5 every 3-4 days. For differentiation to an adipocyte-like phenotype, confluent cells were maintained in culture medium for $48 \mathrm{~h}$ before changing to differentiation medium (DMEM with $4.5 \mathrm{~g} / \mathrm{L}$ glucose, supplemented with $10 \%$ fetal calf serum (FCS) (Thermo Fisher, no. 16000), 1\% PEST, $1.1 \mathrm{~g} / \mathrm{L}$ 3-isobutyl-1methlyxanthine (IBMX), $0.5 \mu \mathrm{M}$ dexamethasone, and $1.7 \mu \mathrm{M}$ insulin. At day 3 of postinduction, the medium was changed to adipocyte maintenance medium (DMEM with $4.5 \mathrm{~g} / \mathrm{L}$ glucose containing $10 \%$ FCS, $1 \%$ penicillin-streptomycin (PEST), and $1.7 \mu \mathrm{M}$ insulin) and replaced every second day. ${ }^{23}$ Experiments were carried out at days 8-10. For PL induction, 3T3-L1 cells were grown in culture medium as above but supplemented with 5-20 $\mu \mathrm{M}$ propranolol or 5-50 $\mu \mathrm{M}$ amiodarone. ${ }^{24}$ The accumulation of PLs depended on the inducing compound concentration (Figure S3) and length of induction time (Figure S4). The largest difference in PL concentration compared to wild-type cells was found after $48 \mathrm{~h}$ of induction with $20 \mu \mathrm{M}$ propranolol. At this concentration, the impact of PLs could be studied without causing the large morphological changes characteristic of manifest phospholipidosis. Therefore, these were the conditions used throughout this work.

Fluorescence-Based Quantification and Imaging of NL and PL Content. Cells were grown in black, clear-bottom 96-well plates under the different culture conditions mentioned above. NLs were stained with AdipoRed Adipogenesis Assay Reagent (Lonza) according to the manufacturer's instructions. Briefly, cells were washed twice with prewarmed PBS, and each well was filled with $200 \mu \mathrm{L}$ of PBS. Upon addition of $5 \mu \mathrm{L}$ of AdipoRed reagent, the plate was placed for $10 \mathrm{~min}$ at $37^{\circ} \mathrm{C}$. Fluorescence was measured at $485 \mathrm{~nm}(\mathrm{ex}) / 520 \mathrm{~nm}(\mathrm{em})$ at two independent occasions.

PLs were labeled using the fluorescent PL N-(7-nitrobenz-2oxa-1,3-diazol-4-yl)-1,2-dihexadecanoyl-sn-glycero-3-phosphoethanolamine (NBD-PE) (Thermo Fisher, no. N-360), which was added to the culture medium at $50 \mu \mathrm{M}$ for $48 \mathrm{~h}$. Cells were washed twice with prewarmed PBS prior to measurement at $485 \mathrm{~nm}(\mathrm{ex}) / 529 \mathrm{~nm}(\mathrm{em})$. All values were normalized for cell number using the signal $(355 \mathrm{~nm}(\mathrm{ex}) / 520 \mathrm{~nm}(\mathrm{em}))$ of the nuclear stain Hoechst $3342(5 \mu \mathrm{g} / \mathrm{mL}$ in PBS) in representative wells.

Confocal images of lipid cellular distribution were obtained with a Zeiss LSM 700 confocal with a Plan-Apochromat 20×/0.8 objective. Instrument settings were identical for each series of images to ensure accurate comparison of the fluorophore staining. The images were analyzed using the Zen 2.1 SP1 software (Carl Zeiss, version 12.0.0.0). The specificity of the stains was confirmed by comparison to control stains with similar staining pattern (Figure S2).

Lysosomal Staining. All stains were from Thermo Fisher and used according to the manufacturer's instructions. Lyso Tracker Red (LTR) DND-99 was used for staining of lysosomes, wheat germ agglutinin for cell membranes, and DAPI for nuclei. After staining, samples were fixed with $4 \%$ formaldehyde for $15 \mathrm{~min}$ and mounted overnight with ProLong Gold Antifade Mountant. Images were captured using a Zeiss LSM 710 with a Plan-Apochromat 40×/1.3 Oil DIC M27 objective. The specificity of the lysosomal stain was verified by observing disappearance of the staining upon addition of $100 \mu \mathrm{M}$ of the $\mathrm{pH}$-gradient disrupting drug, chloroquine, to the staining solution.

Enzymatic Quantification of PL Content. The PL content of cell homogenates was quantified at two independent occasions using the WAKO LabAssay Phospholipid-Choline Oxidase/DAOS method according to the manufacturer's instructions (Nordic Biolabs). Briefly, $2 \mu \mathrm{L}$ of the cell homogenate used for cellular binding studies (see below) and $300 \mu \mathrm{L}$ of color reagent were added to a 96-well black, clear-bottom plate, incubated at $37^{\circ} \mathrm{C}$ for $5 \mathrm{~min}$. Absorbance was measured at $600 \mathrm{~nm}$. A calibration curve was established using the standards provided in the kit.

Proteomics. For global proteomics, the adherent cells were washed twice with PBS, harvested with a cell scraper, and pelleted. Pellets were stored at $-80{ }^{\circ} \mathrm{C}$ until further analysis. Peptides were prepared using the filter-aided sample preparation protocol by enzymatic digestion with Lys-C and trypsin and analyzed on a QExactive mass spectrometer (Thermo Fisher Scientific) in a label-free shotgun approach, as described elsewhere. ${ }^{25,26}$ Proteins were quantified using the label- and standardfree "Total Protein Approach." ${ }^{26}$ Only proteins quantified with $\geq 3$ unique/razor peptides were statistically analyzed with the tool Perseus $^{27}$ (version 1.5.4.0) [http://www.perseus-framework.org]. 
Pathway analysis was performed using the Database for Annotation, Visualization and Integrated Discovery ${ }^{28}$ (DAVID; version 6.8) [https://david.ncifcrf.gov/]. Venn diagrams were prepared using a web-based tool available at http://bioinformatics.psb. ugent.be.

Intracellular Bioavailability $\left(F_{\mathrm{ic}}\right) . F_{\mathrm{ic}}$ was calculated using eq 1

$$
F_{\mathrm{ic}}=\mathrm{Kp} \cdot f_{\mathrm{u}, \mathrm{cell}}
$$

where $\mathrm{Kp}$ is the intracellular compound accumulation at steady state, and $f_{\mathrm{u}, \mathrm{cell}}$ is the intracellular fraction of unbound compound. These were determined as previously described and summarized in the following sections. ${ }^{2}$

Intracellular Compound Accumulation at Steady State (Kp). Cells were cultured as described in the cell culture section. All experiments were performed in 96-well plates. $\mathrm{Kp}$ was measured at $100 \%$ confluence or after full differentiation of the cells. Briefly, cells were washed twice with prewarmed Hank's buffered salt solution (HBSS), and $200 \mu \mathrm{L}$ of $0.5 \mu \mathrm{M}$ drug solution in HBSS was added. An incubation time of $45 \mathrm{~min}$ was found to be sufficient to reach equilibrium for a diverse compound set across all evaluated cell types (Figure S5) and was therefore used in subsequent experiments. A sample of the incubation medium was removed and diluted 10-fold with a mixture of acetonitrile and water $(60: 40)$ containing $50 \mathrm{nM}$ warfarin (internal standard) for determination of $C_{\text {medium }}$ by LC-MS/MS (Table S16). The remaining supernatant was removed by aspiration, and the cells were washed twice with ice-cold PBS. Intracellular compound was extracted using an acetonitrile $/ \mathrm{H}_{2} \mathrm{O}(60: 40)$ solution containing an internal standard for the determination of the amount of drug in the cells $\left(A_{\text {cell }}\right)$. Protein content was quantified using the BCA assay in representative wells to calculate the cellular volume $\left(V_{\text {cell }}\right)$, assuming $6.5 \mu \mathrm{L} / \mathrm{mg}$ protein (Figure $\mathrm{S} 8$ and ref 29 ). Finally, $\mathrm{Kp}$ was calculated using eq 2

$$
\mathrm{Kp}=\frac{A_{\text {cell }} / V_{\text {cell }}}{C_{\text {medium }}}
$$

All experiments were performed in triplicate, and reproducibility was confirmed by repeating the experiment on up to four separate occasions.

Binding of Drug to Cell Homogenates $\left(f_{u, h o m}\right)$ and Intracellular Fraction of Unbound Compound ( $\left.f_{\mathrm{u}, \mathrm{cell}}\right)$. $f_{\mathrm{u}, \text { cell }}$ was measured in cassette mode as previously described. ${ }^{30}$ Up to eight compounds were pooled in each dialysis chamber to increase throughput. ${ }^{30}$ In addition to our previously published comparisons, cassette- and single-dosing equivalency was confirmed here for three representative compounds from our data set (Figure S7, Panel A). Further control experiments showed that the effect of DMSO (present in stock solutions) and chloroquine (used to neutralize the $\mathrm{pH}$ gradient of endolysosomal compartments in the $\mathrm{Kp}$ experiments with intact cells $)^{2}$ did not alter $f_{\mathrm{u}, \text { cell }}$ significantly (Figure S7, panels B and C). Briefly, 10 million cells $/ \mathrm{mL}$ were suspended in HBSS and homogenized on ice by sonication (VCX 750 Sonicator, $3 \mathrm{~mm}$ probe, $20 \%$ intensity, $10 \mathrm{~s}$ ). Compounds were added to the cell homogenate at a final concentration of $0.5 \mu \mathrm{M}$ each, and the spiked homogenate was transferred to the dialysis chamber. Atorvastatin and lopinavir were included in each experiment as controls for low- and high-binding, respectively. HBSS was added to the receiver compartment. Samples of spiked cell homogenates were kept at 4 and $37^{\circ} \mathrm{C}$ for the duration of the experiment as stability controls (only samples with recoveries of $>70 \%$ of the initial compound were included). After dialysis for $4 \mathrm{~h}$ at $37{ }^{\circ} \mathrm{C}$ and $900 \mathrm{rpm}$ in the Rapid Equilibrium Dialysis device (Thermo Fisher), the unbound fraction in the cell homogenate $\left(f_{\mathrm{u}, \mathrm{hom}}\right)$ was determined according to eq 3

$$
f_{\text {u,hom }}=\frac{C_{\text {buffer }}}{C_{\text {hom }}}
$$

The fraction of unbound compound in the cell $\left(f_{\mathrm{u}, \text { cell }}\right)$ was calculated by correcting for homogenate dilution according to a Langmuir binding isotherm model (eq 4)

$$
f_{\mathrm{u}, \text { cell }}=\frac{1}{D \cdot\left(1 / f_{\mathrm{u}, \mathrm{hom}}-1\right)+1}
$$

where the dilution constant $D$ was calculated using eq 5, assuming the $V_{\text {cell }}$ to be equal to $6.5 \mu \mathrm{L} / \mathrm{mg}$ protein (Figure S8 and ref 29).

$$
D=1 / V_{\text {cell }}
$$

Membrane Affinity and Fraction Unbound to Lipids: MA and $f_{\text {u,lipid }}$. The affinity of the compounds to pure phosphatidylcholine membranes was determined using the TRANSIL Intestinal Absorption Kit, according to the manufacturer's instructions (Sovicell, TMP-0100-2096). Membrane affinity was determined at six different concentrations of PL-coated silica beads at a fixed concentration of the test compound. After incubation for $15 \mathrm{~min}$ at room temperature and shaking at $1000 \mathrm{rpm}$, the samples were centrifuged, and the drug concentration in the supernatant $\left(C_{\text {buffer }}\right)$ was quantified by LC-MS/MS (Table S16). Nonspecific binding to assay plastic was determined in wells containing only buffer. Membrane affinity is defined as described in eq 6

$$
\text { membrane affinity }=\frac{C_{\text {membrane }}}{C_{\text {buffer }}}
$$

and was calculated according to instructions in the TRANSIL Intestinal Absorption Kit.

The fraction unbound to pure phosphatidylcholine (PC), $f_{\mathrm{u}, \mathrm{PC}}$ was derived as follows (eq 7)

$$
f_{\mathrm{u}, \mathrm{PC}}=\frac{C_{\text {buffer }}}{C_{\text {buffer }}+C_{\text {membrane }}}
$$

Prediction of $\boldsymbol{f}_{\mathrm{u} \text {,hom }} \cdot f_{\mathrm{u} \text {,hom }}$ was predicted based on $f_{\mathrm{u}, \mathrm{PC}}$ (from eq 7), using the dilution eq (eq 4) to scale the extent of binding from the pure PL system to the conditions in cell homogenates

$$
f_{\mathrm{u}, \text { hom,pred }}=\frac{1}{D \cdot\left(1 / f_{\mathrm{u}, \mathrm{PC}}-1\right)+1}
$$

where the dilution factor $D$ was optimized with the Solver Microsoft Excel add-in (version 14.0) by minimizing the sum of the squared prediction errors.

Statistical Analysis. All statistical analyses (Wilcoxon matched-pairs signed rank tests, $t$ tests with multiple test correction, Bonferroni-Dunn method) were performed in GraphPad Prism (version 7.0).

\section{RESULTS}

Development and Characterization of Cellular Systems. To study the impact of neutral lipids (NL) and phospholipids (PL) on $F_{\mathrm{ic}}$ we established cellular systems of 3T3-L1 

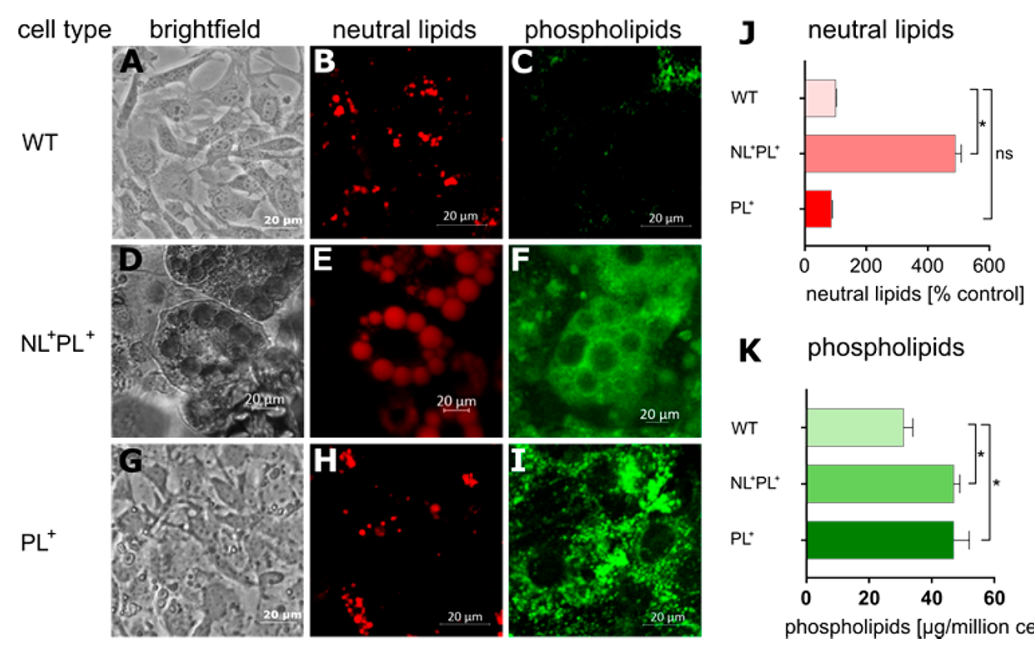

L proteomics - identified proteins

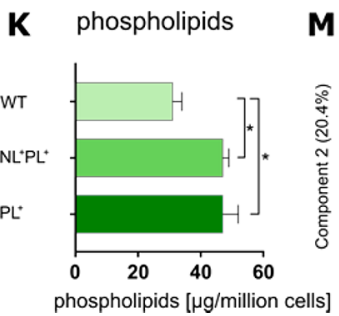

M proteomics - PCA cell types

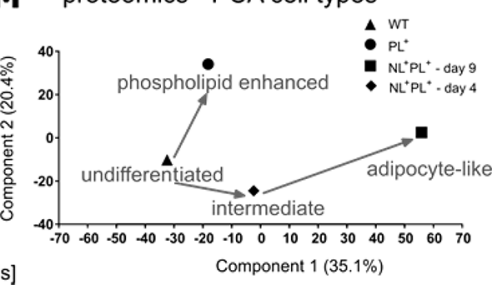

Figure 1. Three cells models in this study. 3T3-L1 mouse fibroblasts were treated to produce adipocyte-like $\left(\mathrm{NL}^{+} \mathrm{PL}{ }^{+}\right.$cells; $\left.\mathrm{D}-\mathrm{F}\right)$ and $\mathrm{PL}-\mathrm{enhanced}$ $\left(\mathrm{PL}^{+}\right.$cells; $\left.\mathrm{G}-\mathrm{I}\right)$ cells. These were compared with untreated wild-type cells (WT cells; A-C) for lipid content and global protein expression. Brightfield (A,D,G); AdipoRed (NL staining: panels B,E,H); and NBD-PE (PL staining; panels C,F,I) images are shown. NL (panel K) and PL (panel J) content was determined in the different cellular models $\left({ }^{*} p<0.05\right)$. A total of 5911 proteins were identified in a global proteomics study (L). A principal component analysis (PCA) was performed based on the concentrations of the quantifiable proteins ( $\geq 3$ unique/razor peptides, $n=3755)(\mathrm{M}$, loading plot available in Figure S14).

cells with increased NL and PL content. We then compared the lipid-enriched cell models with the wild-type (WT) controls containing normal amounts of lipids.

Lipid Content. WT cells had very few and small NL droplets. These cells also lacked noticeable accumulation of fluorescently labeled PL (NBD-PE), indicating a normal PL homeostasis (Figure 1A-C). After differentiation of the WT cells into adipocyte-like cells, we observed a 5-fold increase in staining of NLs (Figure $1 \mathrm{~J}$ ) and a 1.5 -fold increase in PL content (Figure $1 \mathrm{~K}$ ) these cells are therefore named $\mathrm{NL}^{+} \mathrm{PL}^{+}$cells. After treatment of the WT cells with the phospholipidosis-inducing cationic drug propranolol, the NL content remained unchanged (Figure $1 \mathrm{H}, \mathrm{J}$ ), but the PL content increased 1.5-fold (Figure 1I, K)-these cells are therefore named $\mathrm{PL}^{+}$cells.

Proteomics. We characterized the three cell models using mass spectrometry-based global proteomics. A total of 5911 proteins were identified (Figure 1L), of which 3755 were confidently quantified. These numbers were in line with previously published proteomes of 3T3-L1 cells in studies with comparable quantification techniques. ${ }^{31-34}$ Of these 3755 quantified proteins, 472 were differentially expressed in $\mathrm{NL}^{+} \mathrm{PL}^{+}$cells compared to the WT cells (Figure S13). Pathway analysis showed that these 472 proteins were involved in metabolic processes, glycolysis and gluconeogenesis, lipid storage and homeostasis, and fatty acid turnover (Figure S13). As expected, the adipocyte-like $\mathrm{NL}^{+} \mathrm{PL}^{+}$cells showed up to 100 -fold higher expression of well-known markers of adipocyte differentiation (e.g., Lipe, Fabp4), lipid droplet associated proteins (e.g., Plin 1, Abhd5), and proteins involved in fatty acid metabolism (e.g., Lpl, Acsl1) ${ }^{31,32}$ (Figure S14). Cells harvested at an intermediate stage of differentiation (day 4 instead of day 9) showed an intermediate proteomic profile in a principal component analysis (Figures $1 \mathrm{M}$ and S14).

The proteome of $\mathrm{PL}^{+}$cells showed only minor differences of no statistical significance, compared to the WT cells (Figures $1 \mathrm{M}$ and S13). Cells with morphological signs of phospholipidosis in vitro typically show upregulation of proteins involved in, e.g., lysosomal activity, cholesterol biosynthesis (HMG-CoA reductase and synthase), and lipid metabolism. ${ }^{35}$ This was not the case in $\mathrm{PL}^{+}$cells, suggesting that these cells-induced for only 2 days-were at an early stage of phospholipidosis.

Proteins involved in ADME processes, including transporters and metabolic enzymes, have previously been shown to alter $F_{\text {ic }}{ }^{7}$ We therefore analyzed their cellular concentrations. A total of 110 ATP-binding cassette $(\mathrm{ABC})$ proteins and solute carrier family (SLC) transporters were identified in the global proteomic analysis (see Supporting Information), of which 15 are known to impact drug disposition; none of these were significantly altered by the differentiation procedures. Likewise, the expression of the nine identified cytochrome P450 (CYP) enzymes was not significantly altered. From these results, we expect that these drug transporters and drug metabolizing enzymes did not influence the differences in $F_{\text {ic }}$ of the three cell lines in this study.

Cell Volume. We also investigated if the protein mass per cell volume remained constant for the three cell types, as is generally assumed for mammalian cells. ${ }^{36,37}$ We determined the cell number, total protein content, and average cell diameter for each cell type after culture in 24-well plates. The difference in cellular volume per protein mass was minimal (6.5 to $7.1 \mu \mathrm{L} / \mathrm{mg}$ protein) (Figure S8).

Impact of Lipids on Intracellular Bioavailability. A set of 23 chemically diverse drugs, representative of the chemical space of registered drugs and differing in charge, lipophilicity, polarity, and size (Figure S1), was selected to evaluate the differences in $F_{\mathrm{ic}}$ in the three cell types. $F_{\mathrm{ic}}$ is the product of steadystate cellular uptake $(\mathrm{Kp})$ and unbound drug fraction in cells $\left(f_{\text {u,cell }}\right)$ (Table 1; Figure 2).

Effect of Lipids on $F_{i c} . F_{\text {ic }}$ was generally lower in $\mathrm{NL}^{+} \mathrm{PL}^{+}$ cells $(p=0.01$, Wilcoxon matched-pairs signed rank test, $70 \%$ of the compounds) and $\mathrm{PL}^{+}$cells ( $p=0.02,80 \%$ of the compounds) than in the WT cells. $F_{\text {ic }}$ in WT and $\mathrm{PL}^{+}$cells (that have intact, acidic lysosomes) was dependent on the charge of the compounds (difference between charge classes of drugs in WT cells $p=0.004$ and in $\mathrm{PL}^{+}$cells $p=0.003$, Kruskal-Wallis test), with higher values for cations and lower values for anions as compared to neutral compounds in both cell types. This observation likely reflected charge-dependent 

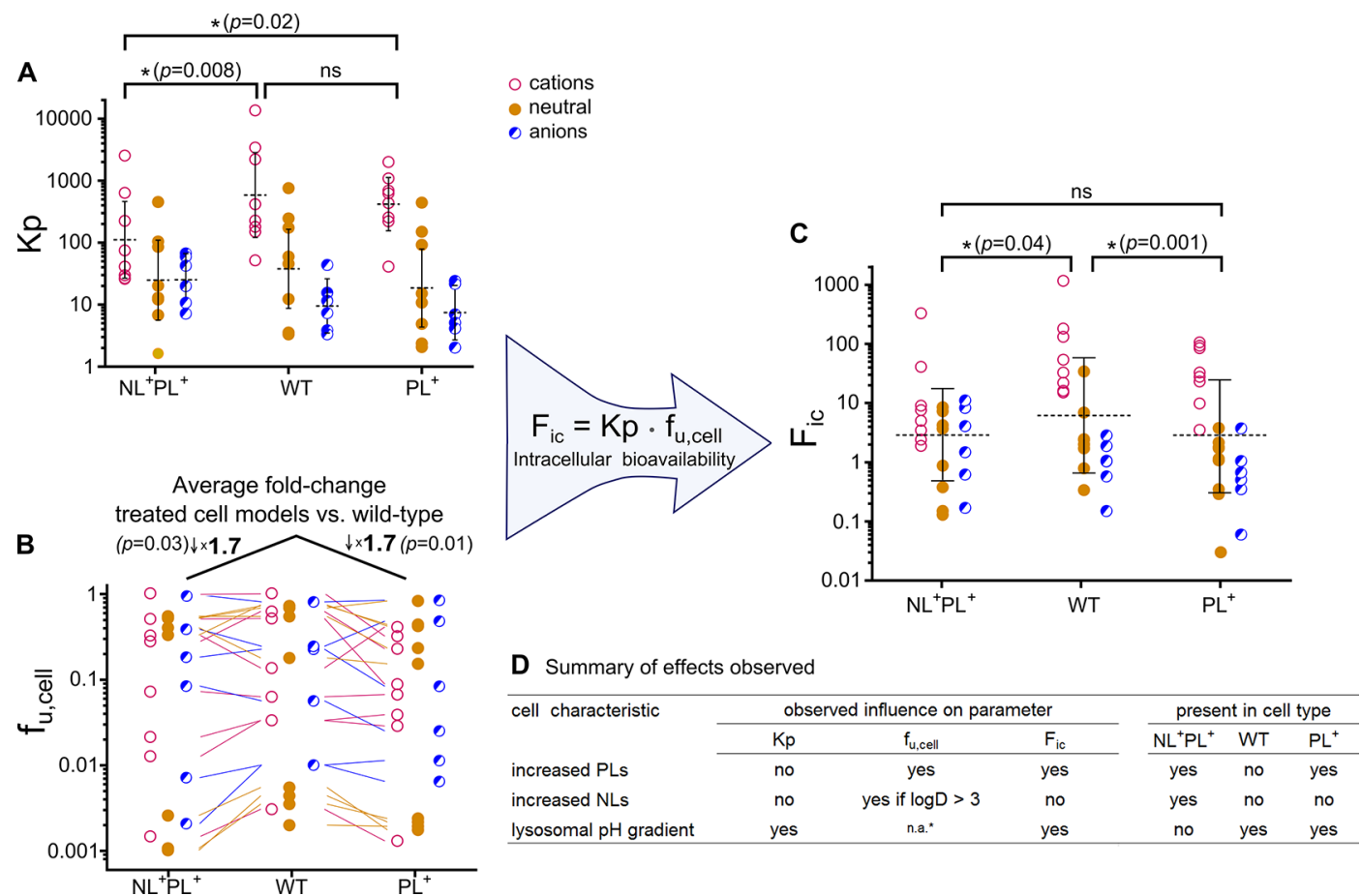

Figure 2. Intracellular bioavailability $\left(F_{\mathrm{ic}}\right)$ of the 23 investigated compounds in the three cell models. (A) Kp represented per cell type and per charge of the compounds. Differences were assessed separately for each drug class. A significant decrease in accumulation of cationic compounds in the $\mathrm{NL}^{+} \mathrm{PL}^{+}$cells compared to the WT $(p=0.008)$ and $\mathrm{PL}^{+}(p=0.02)$ cells was observed. (B) Comparison of $f_{\mathrm{u} \text {,cell }}$ between the three cell types. On average, $f_{\mathrm{u}, \text { cell }}$ was significantly lower in both treated cell models by a factor of 1.7 (C) Overall, the resulting $F_{\mathrm{ic}}$ was significantly lower in the two treated cell models compared to WT cells $\left(p=0.04\right.$ and 0.001 for $\mathrm{NL}^{+} \mathrm{PL}^{+}$and $\mathrm{PL}^{+}$cells, respectively). (D) Summary of the parameters studied and their observed influence on Kp, $f_{\mathrm{u}, \text { cell, }}$ and $F_{\mathrm{ic}}{ }^{*}$ n.a. = not applicable (ruptured lysosomes upon homogenization, Figure S7). Note: The same figure with individually labeled data points is available in S17.

lysosomal trapping of cationic drugs and limited cell penetration of anionic drugs. ${ }^{2,7,38}$ However, the charge dependence was less pronounced in $\mathrm{NL}^{+} \mathrm{PL}^{+}$cells (that have lysosomes with neutralized $\mathrm{pH})(p=0.256$, Kruskal-Wallis test). Accordingly, lysosomal staining with the acidotropic probe Lyso Tracker Red was reduced in the $\mathrm{NL}^{+} \mathrm{PL}^{+}$cells, while WT and $\mathrm{PL}^{+}$cells presented normal, distinct lysosomal staining (Figure 3A). When the cells were incubated with the lysosomal $\mathrm{pH}$-elevating drug chloroquine, the lysosomal staining disappeared in all three cell types. In addition, accumulation of the lysosomotropic drug alprenolol decreased in the chloroquine-treated $\mathrm{WT}$ and $\mathrm{PL}^{+}$ cells to similar levels as in untreated $\mathrm{NL}^{+} \mathrm{PL}^{+}$cells (Figure $3 \mathrm{~B}$ ). In the same experiment, $\mathrm{Kp}$ values of the neutral and anionic drugs were unaffected (Figure 3B). Taken together, these results suggest that lysosomal $\mathrm{pH}$ is increased in $\mathrm{NL}^{+} \mathrm{PL}^{+}$cells, leading to lower accumulation of basic drugs in low-pH compartments. The decrease in $\mathrm{Kp}$ in these cells (Figure $2 \mathrm{~A}$ ) was therefore not attributed to the presence of lipids. Note that the differences in $\mathrm{Kp}$ of neutral drugs in $\mathrm{PL}^{+}$vs WT cells reached statistical significance. This observation was not explained merely by the lipophilicity of the drugs. Further evaluation in separate studies may provide an explanation but is beyond the scope of this manuscript. Kp reflects the sum of all processes leading to the net drug accumulation in cells, such as passive and active transport, metabolism and unspecific binding.

Effects of Lipids on $f_{u \text {,cell }}$. To study the effect of lipids on $f_{\mathrm{u}, \mathrm{cell}}$, we compared the values of the differentiated cell models and the WT cells. As previously observed, ${ }^{2,39} f_{\text {u,cell }}$ decreased with $\log \mathrm{D}_{7.4}$ (Figure S9a). Our compounds displayed, on average, a 1.7-fold higher binding to both $\mathrm{NL}^{+} \mathrm{PL}^{+}$and $\mathrm{PL}^{+}$cells than WT cells, resulting in a lower $f_{\text {u,cell }}$ for the two treated cell types (Figure 2B). This increase in binding was in good agreement with the 1.5-fold average increase in PL content in both $\mathrm{NL}^{+} \mathrm{PL}^{+}$and $\mathrm{PL}^{+}$cells (Figure $1 \mathrm{~K}$ ). Preliminary experiments using $\mathrm{PL}^{+}$cells with a different $\mathrm{PL}$ content supported these findings (Figure S4). Although the average change was similar in both treated cell types, cationic drugs were somewhat more affected in the $\mathrm{PL}^{+}$cells, and neutral compounds were more affected in the $\mathrm{NL}^{+} \mathrm{PL}^{+}$cells; in both cases, there was a 2.1 -fold increase relative to the WT cells (Figure $1 \mathrm{~K}$ ). In contrast, the approximately 5 -fold increase in NLs in the $\mathrm{NL}^{+} \mathrm{PL}^{+}$cells did not seem to quantitatively affect $f_{\mathrm{u}, \text { cell }}$ for the data set overall, although a trend of increased binding was observed for the highly lipophilic compounds $(\log \mathrm{D}>3$, Figure S9b). Together, these results suggest that PLs are a major determinant of the unbound drug fraction inside the cell for most compounds.

Contribution of PLs to Cellular Binding. To further investigate the impact of PLs on $f_{\mathrm{u}, \mathrm{cell}}$, we measured binding of the same compound set to phosphatidylcholine-coated beads (Figure S10). These beads are designed for determining membrane binding, and the PLs are arranged in a bilayer conformation on the bead surface. ${ }^{40}$ We scaled the unbound fraction measured in the phosphatidylcholine bead system $\left(f_{\mathrm{u}, \mathrm{PC}}\right)$ to $f_{\mathrm{u}, \text { cell }}$ with the Langmuir isotherm model that is commonly used to account for dilution in measurements of drug binding to tissues $^{2,41}$ (Figure 4A). Our predictions of $f_{\mathrm{u}, \text { cell }}$ correlated well with the $f_{\mathrm{u} \text {,cell }}$ from the cell homogenates. A larger experimental error was observed for compounds with $f_{\text {u,cell }}$ close to 1 (Figure 4B,C). This is explainable by the considerably higher PL concentration in the cell-free system, which resulted in a higher resolution for low-binding compounds in this system than that obtained in the cellular homogenates. ${ }^{7}$ The dilution 
A

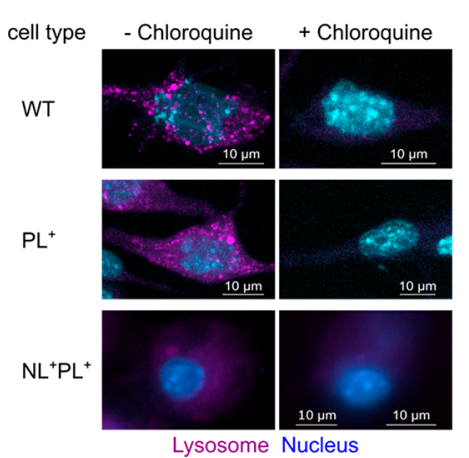

\section{B}

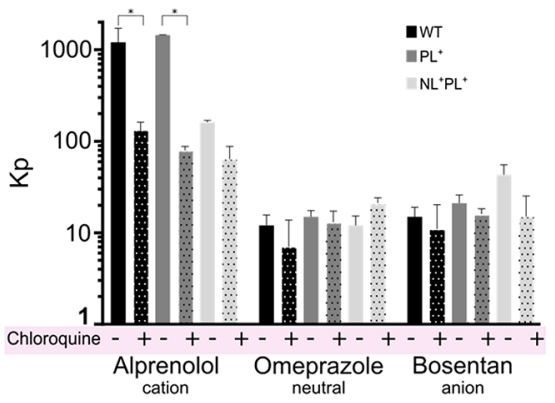

Figure 3. Effect of chloroquine on the accumulation of one representative compound from each charge class in the three cell systems. (A) Acidic compartments (shown in purple) were not detected in $\mathrm{NL}^{+} \mathrm{PL}^{+}$cells. Disruption of the lysosomal $\mathrm{pH}$ gradient with chloroquine resulted in loss of staining in all cell types. Images with additional staining of the cellular membrane are available in Figure S6. (B) Kp of drugs representing the three charge classes in all three cells types, in the presence and absence of chloroquine. Chloroquine reduced the Kp of the cation alprenolol in WT and $\mathrm{PL}^{+}$cells $\left(p<0.0001\right.$, Bonferroni-Dunn method) but did not significantly affect the accumulation in $\mathrm{NL}^{+} \mathrm{PL}^{+}$cells $(p=0.32)$. Accumulation of the neutral and anionic compounds, omeprazole and bosentan, was not affected by chloroquine (all $p$-values $>0.7$ ).

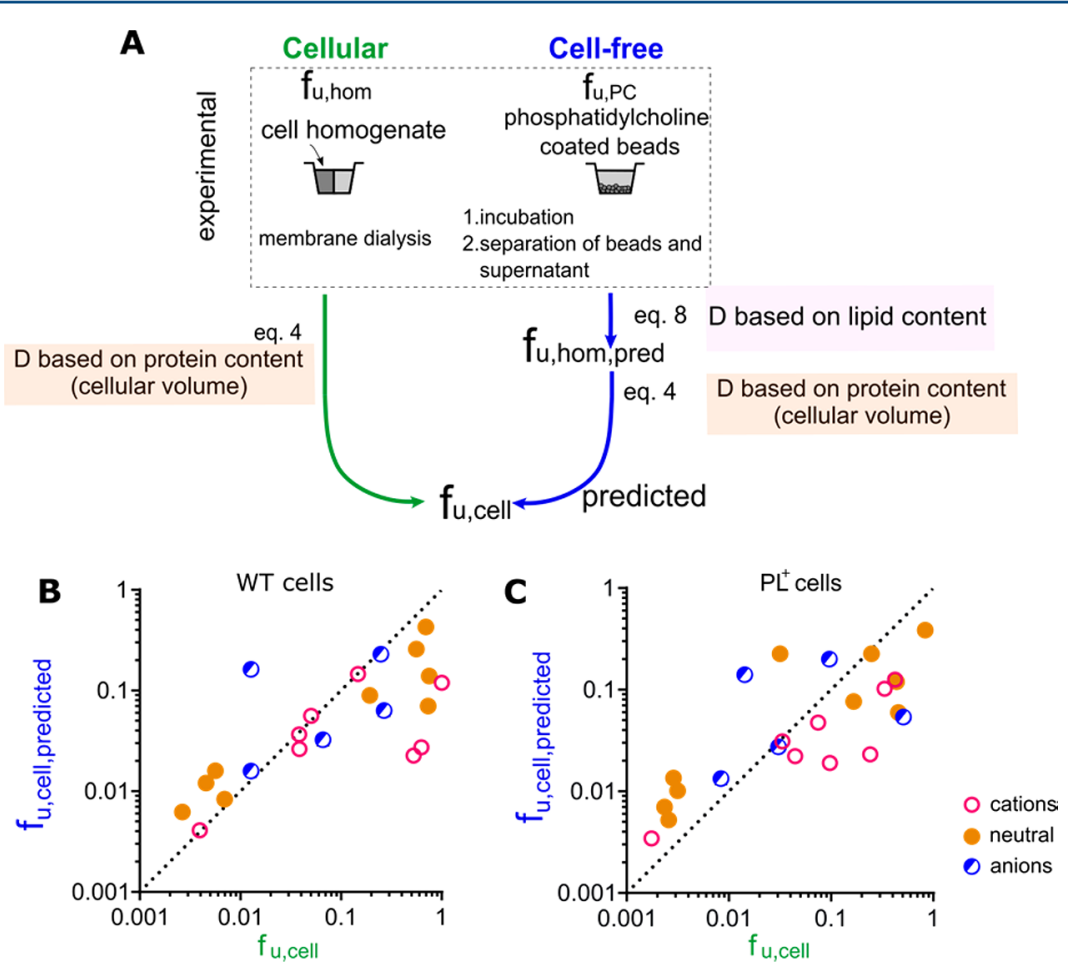

Figure 4. Determination of $f_{\mathrm{u}, \text { cell }}$ for cell homogenates and cell-free PL beads. (A) Experimental procedure for determination of $f_{\mathrm{u}, \text { cell }}$ based on experimental measurement of $f_{\mathrm{u} \text {,hom }}$ (left, green) or $f_{\mathrm{u}, \mathrm{PC}}$ (right, blue). (B, C) Correlations between $f_{\mathrm{u}, \text { cell }}$ values measured by the standard cellular assay and the corresponding parameter predicted from the cell-free assay by accounting for lipid and protein dilution. Correlation coefficients were 0.73 and 0.82 for WT and $\mathrm{PL}^{+}$cells, respectively. The predicted values are given in Figure S11.

factors for the WT and $\mathrm{PL}^{+}$cells differed by 1.3 (Figure S11), which is close to the 1.5-fold difference in PL content between the two cell types (Figure $1 \mathrm{~K}$ ). In conclusion, it seems that $f_{\mathrm{u}, \text { cell }}$ can be predicted from affinities to pure PLs when the lipid content of the cells is known, again suggesting that PLs are the major determinant of intracellular drug binding.

\section{DISCUSSION}

Intracellular bioavailability $\left(F_{\mathrm{ic}}\right)$ is the net result of all factors that influence cellular drug disposition, but the individual contribution of each factor has not yet been studied in detail. In this study, we focused on how cellular lipid content influences $F_{\text {ic }}$. Lipophilic drugs distribute to cellular lipids, ${ }^{16,18,19}$ but the effects on unbound intracellular drug exposure, as described by the $F_{\text {ic }}$ parameter, have not been investigated under controlled and well-characterized cell culture conditions. We chose the 3T3-L1 (mouse fibroblast) cell model for these studies, because it can be grown in different configurations resulting in different lipid compositions. ${ }^{22}$ Our results indicated a strong reversed relationship between the increase in PL content and the equal difference in intracellular fraction of unbound compound $\left(f_{\mathrm{u}, \mathrm{cell}}\right)$. The 5-fold increase in the content of NLs only affected highly lipophilic drugs and did not generally enhance the binding beyond the increases seen when only PL content was induced. Further support for a dominant role of PLs in cellular drug distribution was obtained from a cell-free system containing only phosphatidylcholine, the most abundant PL in 
mammalian cells. ${ }^{44,45}$ There was a strong correlation between the $f_{\mathrm{u} \text {,cell }}$ predicted from binding to the pure PL system and the $f_{\mathrm{u}, \text { cell }}$ measured in cellular homogenates (Figure 4B,C). We speculate that most of the remaining differences between the PL-derived and cellular $f_{\mathrm{u} \text {,cell }}$ are due to real cell membranes having a more complex lipid composition.

Distribution of certain neutral drugs into NL droplets has been reported. ${ }^{46-48}$ It was therefore unexpected that the accumulation of our neutral drugs did not increase in the $\mathrm{NL}^{+} \mathrm{PL}^{+}$cells. Lipid droplets, such as those present in the $\mathrm{NL}^{+} \mathrm{PL}^{+}$cells, contain a core that is filled mainly with NLs such as triacylglycerols and cholesterol esters; these NLs are surrounded by a PL monolayer originating from the endoplasmic reticulum. ${ }^{49,50}$ Amphiphilic drugs are therefore likely to accumulate on the surface of these droplets rather than penetrate into the NL core. While our results do not indicate preferential accumulation of drugs in $\mathrm{NL}$ droplets, it is possible that neutral drugs more lipophilic than those in our study might be.

Initially, we intended to include more compounds with very high $V_{\mathrm{D}}$ (up to $700 \mathrm{~L}$ ) in our data set, since a potential link between tissue uptake and strong lipid binding has been discussed in the literature. ${ }^{16,20}$ However, due to the poor ionization properties of these lipophilic compounds (with resulting low sensitivity in LC-MS/MS analysis), they did not satisfy the quality thresholds. Further studies are required to determine a potential correlation between lipid affinities and (high) $V_{\mathrm{D}}$. Nevertheless, the compound set used in this study was carefully chosen on the basis of a principal component analysis that included 1197 drugs and 334 molecular descriptors (Figure S1). The final compound set covered key properties such as molecular charge (anions, neutral compounds, and cations), $\log D_{7.4}$ (-0.7 to 5.0), PSA (28.2-146.0 $\left.\AA^{2}\right)$, MW (194.2-628.8), and $\mathrm{V}_{\mathrm{D}}(0.13$ to $250 \mathrm{~L})$.

Factors other than lipid composition could also contribute to the differences in $F_{\text {ic }}$ between the three 3T3-L1 cell types. For instance, the divergent morphology of the adipocyte-like $\mathrm{NL}^{+} \mathrm{PL}^{+}$ cells suggested that the global protein expression profiles might also be altered. We therefore investigated the cellular proteomic profiles of the three cell lines.

The protein profile of the $\mathrm{PL}^{+}$cells was not significantly different from that of the WT cells, but the $\mathrm{NL}^{+} \mathrm{PL}^{+}$cells overexpressed typical adipocyte-specific markers ${ }^{31-34}$ (Figures 1L,M, S13, and S14). Interestingly, the adipocyte-specific fatty acid binding protein 4 (Fabp4) was highly upregulated to $422.1 \mathrm{fmol} / \mu \mathrm{g}$ protein in $\mathrm{NL}^{+} \mathrm{PL}^{+}$cells, compared to $2.6 \mathrm{fmol} / \mu \mathrm{g}$ protein in WT cells. Fabp5 was also detected, albeit with only a minor increase in the $\mathrm{NL}^{+} \mathrm{PL}^{+}$cells. Members of the fatty acid binding protein (FABP) family are among the most abundant proteins in the cytosol and have a role in intracellular trafficking as carriers of insoluble lipophilic compounds. ${ }^{51-53}$ FABPs can therefore alter the exposure at targets residing in, for example, the nucleus. Similarly, FABPs in intestinal epithelial cells have been shown to influence the uptake and disposition of drugs in models for intestinal absorption. ${ }^{51}$ FABPs generally bind drugs at a $1: 1$ stoichiometry. ${ }^{53}$ Binding affinity constants of the FABPs are in the micromolar range, with increased affinity toward lipophilic and acidic compounds. ${ }^{51-54}$ However, for a given drug, the affinity to FABPs is generally lower than the affinity to albumin. ${ }^{54}$ In turn, albumin has, on average, lower binding affinities for drugs than do the PLs. ${ }^{40}$ Further, PLs are more abundant than FABPs - in our WT cellular homogenates, we quantified roughly $35000 \mathrm{PL}$ molecules per FABP protein (Figure S15). Together, this supports the general notion in our study that the differences in drug binding in our three cell models were caused mainly by PLs, with intracellular drug protein binding having little impact.

We have previously shown that alterations in the expression of ADME proteins, such as drug transporters and drug metabolizing enzymes, affect the $F_{\mathrm{ic}}{ }^{7}$ We therefore analyzed whether the expression of ADME-related proteins differed in our cell lines. However, no significant differences were seen, indicating a limited impact of these proteins on $F_{\text {ic }}$ in our study.

However, one significant finding was the absence of a $\mathrm{pH}$ gradient between cytosol and lysosomes in the $\mathrm{NL}^{+} \mathrm{PL}^{+}$cells (Figure 3A). Lipophilic cationic drugs are well-known to be trapped in low $\mathrm{pH}$ compartments such as the lysosomes, and this was observed in the $\mathrm{PL}^{+}$and WT cells but not in the $\mathrm{NL}^{+} \mathrm{PL}^{+}$. The increased lysosomal permeability in hypertrophic adipocytes reported in vivo supports our results. ${ }^{55}$ If this increased permeability exceeds the capacity of the endolysosomal proton accumulation, it could explain the absence of the $\mathrm{pH}$ gradient in our $\mathrm{NL}^{+} \mathrm{PL}^{+}$cells. A comparable endolysosomal permeability defect is seen in cells where the $\mathrm{Cl}^{-} / \mathrm{HCO}_{3}{ }^{-}$exchanger Slc4a2 is depleted. ${ }^{56}$ Interestingly, this transporter was down-regulated in our $\mathrm{NL}^{+} \mathrm{PL}^{+}$cells, but further investigations are required to establish the role of Slc4a2 for endolysosomal integrity.

Finally, we confirmed a constant ratio between cellular volume and total cellular protein mass. Sensitivity analyses showed that the observed small differences between the cell lines (ranging from 6.5 to $7.1 \mu \mathrm{L} / \mu \mathrm{g}$ protein) did not significantly influence our $F_{\text {ic }}$ calculations. Thus, changes in cellular volume per protein mass could be eliminated as a confounding factor in our studies.

In conclusion, our results indicate that PLs play a major role in determining $f_{\mathrm{u}, \text { cell }}$. Further, elevated endolysosomal $\mathrm{pH}$, as observed in the adipocytes, also influences $F_{\text {ic }}$, while other potentially contributing factors-such as drug binding FABPs, drug transporters, and drug metabolizing enzymes-did not confound our observations. Finally, purified PL beads may be used as a simple and cell-free alternative for high-throughput prediction of $f_{\mathrm{u}, \text { cell }}$.

\section{ASSOCIATED CONTENT}

\section{S Supporting Information}

The Supporting Information is available free of charge on the ACS Publications website at DOI: 10.1021/acs.molpharmaceut.8b00064.

S1: Compound selection-principal component analysis chemical space; S2: stains for PLs and NLs; S3: accumulation of lipids in 3T3-L1 cells by use of propranolol or amiodarone; S4: intermediate PL content results in intermediate binding; S5: time to equilibrium for $\mathrm{Kp}$; S6: lysosomal staining, including membrane stain; S7: $f_{\mathrm{u} \text {,cell }}$ : cassette-dosing and addition of DMSO or chloroquine; S8: evaluation of protein constant for different cell types; S9: $\log D_{7.4}$ vs $f_{\mathrm{u}, \text { cell }}, \mathrm{Kp}$, and $F_{\mathrm{ic}} ; \mathrm{S} 10$ : membrane affinity values measured using the TRANSIL membrane affinity kit; S11: predicted $f_{\mathrm{u} \text {,hom }}$ and $f_{\mathrm{u} \text {,cell }}$ values; S12: partition ratios calculated from $f_{\mathrm{u}, \text { cell }}$ values; S13: proteomics: volcano plot analysis and pathway analysis; S14: proteomics: principal component analysis; S15: number of FABP and PL molecules present in one dialysis chamber; S16: LC-MS/MS methods; S17: Figure 2 with individually labeled data points; the mass spectrometry proteomics data have been deposited to the ProteomeXchange Consortium via the PRIDE partner repository with the data set identifier PXD008994 (PDF) 


\section{AUTHOR INFORMATION}

\section{Corresponding Author}

*Mailing Address: Per Artursson, PhD, Professor in Dosage Form Design, Department of Pharmacy, Uppsala University, Box 580, SE-751 23 Uppsala, Sweden; E-mail: per.artursson@farmaci. uu.se; Phone: +46-18 47144 71; Fax: +46-18 4714471.

\section{ORCID $\odot$}

Andrea Treyer: 0000-0002-4533-7761

André Mateus: 0000-0001-6870-0677

Jacek R Wiśniewski: 0000-0002-8452-5095

Pär Matsson: 0000-0002-9094-2581

\section{Funding}

Financial support was provided by ARIADME, a European FP7 ITN Community's Seventh Framework Program, grant no. 60751 (Andrea Treyer); a $\mathrm{PhD}$ training grant from Fundação para a Ciência e Tecnologia, grant no. SFRH/BD/68304/2010 (André Mateus); the Swedish Fund for Research without Animal Experiments, Magnus Bergvall Foundation, Åke Wiberg Foundation (Pär Matsson); and the Swedish Research Council, grants no. 2822 and 2017-01951 (Per Artursson). The proteomic analyses were funded by the Max-Planck Society for the Advancement of Science by the German Research Foundation (DFG/Gottfried Wilhelm Leibniz Prize) (Jacek R. Wiśniewski).

Notes

The authors declare no competing financial interest.

\section{ACKNOWLEDGMENTS}

We thank Prof. Margareta Hammarlund-Udenaes for valuable discussions. Technical support for imaging with structure illumination microscopy (SIM) was provided by Dr. Georgiy Khodus. We thank Sovicell GmbH for providing the Membrane Affinity kit. We thank Prof. Magnus Ingelman-Sundberg for advice on differentiation of 3T3-L1 cells. We thank Simulations Plus for access to the ADMET Predictor software and ChemAxon for access to the JChem Suite.

\section{REFERENCES}

(1) Mateus, A.; Gordon, L. J.; Wayne, G. J.; Almqvist, H.; Axelsson, H.; Seashore-Ludlow, B.; Treyer, A.; Matsson, P.; Lundback, T.; West, A.; Hann, M. M.; Artursson, P. Prediction of intracellular exposure bridges the gap between target- and cell-based drug discovery. Proc. Natl. Acad. Sci. U. S. A. 2017, 114, E6231.

(2) Mateus, A.; Matsson, P.; Artursson, P. Rapid measurement of intracellular unbound drug concentrations. Mol. Pharmaceutics 2013, 10 (6), 2467-78.

(3) Hallifax, D.; Houston, J. B. Uptake and intracellular binding of lipophilic amine drugs by isolated rat hepatocytes and implications for prediction of in vivo metabolic clearance. Drug metabolism and disposition: the biological fate of chemicals 2006, 34 (11), 1829-36.

(4) Pfeifer, N. D.; Harris, K. B.; Yan, G. Z.; Brouwer, K. L. Determination of intracellular unbound concentrations and subcellular localization of drugs in rat sandwich-cultured hepatocytes compared with liver tissue. Drug Metab. Dispos. 2013, 41 (11), 1949-56.

(5) Keemink, J.; Augustijns, P.; Annaert, P. Unbound ritonavir concentrations in rat and human hepatocytes. J. Pharm. Sci. 2015, 104 (7), 2378-87.

(6) Riccardi, K.; Li, Z.; Brown, J. A.; Gorgoglione, M. F.; Niosi, M.; Gosset, J.; Huard, K.; Erion, D. M.; Di, L. Determination of Unbound Partition Coefficient and in Vitro-in Vivo Extrapolation for SLC13A Transporter-Mediated Uptake. Drug Metab. Dispos. 2016, 44 (10), 1633-42.

(7) Mateus, A.; Treyer, A.; Wegler, C.; Karlgren, M.; Matsson, P.; Artursson, P. Intracellular drug bioavailability: a new predictor of system dependent drug disposition. Sci. Rep. 2017, 7, 43047.
(8) Friden, M.; Bergstrom, F.; Wan, H.; Rehngren, M.; Ahlin, G.; Hammarlund-Udenaes, M.; Bredberg, U. Measurement of unbound drug exposure in brain: modeling of $\mathrm{pH}$ partitioning explains diverging results between the brain slice and brain homogenate methods. Drug metabolism and disposition: the biological fate of chemicals 2011, 39 (3), 353-62.

(9) Riede, J.; Camenisch, G.; Huwyler, J.; Poller, B. Current In Vitro Methods to Determine Hepatic Kpuu: A Comparison of Their Usefulness and Limitations. J. Pharm. Sci. 2017, 106 (9), 2805-2814.

(10) Teuscher, K. B.; Zhang, M.; Ji, H. A Versatile Method to Determine the Cellular Bioavailability of Small-Molecule Inhibitors. J. Med. Chem. 2017, 60 (1), 157-169.

(11) Almqvist, H.; Axelsson, H.; Jafari, R.; Dan, C.; Mateus, A.; Haraldsson, M.; Larsson, A.; Martinez Molina, D.; Artursson, P.; Lundback, T.; Nordlund, P. CETSA screening identifies known and novel thymidylate synthase inhibitors and slow intracellular activation of 5-fluorouracil. Nat. Commun. 2016, 7, 11040.

(12) Funk, R. S.; Krise, J. P. Cationic amphiphilic drugs cause a marked expansion of apparent lysosomal volume: implications for an intracellular distribution-based drug interaction. Mol. Pharmaceutics 2012, 9 (5), 1384-95.

(13) Chien, H. C.; Zur, A. A.; Maurer, T. S.; Yee, S. W.; Tolsma, J.; Jasper, P.; Scott, D. O.; Giacomini, K. M. Rapid Method To Determine Intracellular Drug Concentrations in Cellular Uptake Assays: Application to Metformin in Organic Cation Transporter 1Transfected Human Embryonic Kidney 293 Cells. Drug Metab. Dispos. 2016, 44 (3), 356-64.

(14) Durazo, S. A.; Kadam, R. S.; Drechsel, D.; Patel, M.; Kompella, U. B. Brain mitochondrial drug delivery: influence of drug physicochemical properties. Pharm. Res. 2011, 28 (11), 2833-47.

(15) Nikolaeva, O.; Moiseyev, G.; Rodgers, K. K.; Ma, J. X. Binding to lipid membrane induces conformational changes in RPE65: implications for its isomerohydrolase activity. Biochem. J. 2011, 436 (3), 591-7.

(16) Murakami, T.; Yumoto, R. Role of phosphatidylserine binding in tissue distribution of amine-containing basic compounds. Expert Opin. Drug Metab. Toxicol. 2011, 7 (3), 353-64.

(17) Austin, R. P.; Barton, P.; Mohmed, S.; Riley, R. J. The binding of drugs to hepatocytes and its relationship to physicochemical properties. Drug metabolism and disposition: the biological fate of chemicals 2005, 33 (3), 419-425.

(18) Alakoskela, J. M.; Vitovic, P.; Kinnunen, P. K. Screening for the drug-phospholipid interaction: correlation to phospholipidosis. ChemMedChem 2009, 4 (8), 1224-51.

(19) Nagar, S.; Korzekwa, K. Commentary: nonspecific protein binding versus membrane partitioning: it is not just semantics. Drug Metab. Dispos. 2012, 40 (9), 1649-52.

(20) Rodgers, T.; Jones, H. M.; Rowland, M. Tissue lipids and drug distribution: dog versus rat. J. Pharm. Sci. 2012, 101 (12), 4615-26.

(21) Yata, N.; Toyoda, T.; Murakami, T.; Nishiura, A.; Higashi, Y. Phosphatidylserine as a determinant for the tissue distribution of weakly basic drugs in rats. Pharm. Res. 1990, 7 (10), 1019-1025.

(22) Poulos, S. P.; Dodson, M. V.; Hausman, G. J. Cell line models for differentiation: preadipocytes and adipocytes. Exp. Biol. Med. 2010, 235 (10), 1185-93.

(23) Neve, E. P.; Nordling, A.; Andersson, T. B.; Hellman, U.; Diczfalusy, U.; Johansson, I.; Ingelman-Sundberg, M. Amidoxime reductase system containing cytochrome b5 type B (CYB5B) and MOSC2 is of importance for lipid synthesis in adipocyte mitochondria. J. Biol. Chem. 2012, 287 (9), 6307-17.

(24) Kasahara, T.; Tomita, K.; Murano, H.; Harada, T.; Tsubakimoto, K.; Ogihara, T.; Ohnishi, S.; Kakinuma, C. Establishment of an in vitro high-throughput screening assay for detecting phospholipidosis-inducing potential. Toxicol. Sci. 2006, 90 (1), 13341.

(25) Wisniewski, J. R.; Mann, M. Consecutive proteolytic digestion in an enzyme reactor increases depth of proteomic and phosphoproteomic analysis. Anal. Chem. 2012, 84 (6), 2631-7. 
(26) Wisniewski, J. R.; Rakus, D. Multi-enzyme digestion FASP and the 'Total Protein Approach'-based absolute quantification of the Escherichia coli proteome. J. Proteomics 2014, 109, 322-31.

(27) Tyanova, S.; Temu, T.; Sinitcyn, P.; Carlson, A.; Hein, M. Y.; Geiger, T.; Mann, M.; Cox, J. The Perseus computational platform for comprehensive analysis of (prote)omics data. Nat. Methods 2016, 13 (9), 731-40.

(28) Huang, D. W.; Sherman, B. T.; Lempicki, R. A. Systematic and integrative analysis of large gene lists using DAVID bioinformatics resources. Nat. Protoc. 2009, 4 (1), 44-57.

(29) Gillen, C. M.; Forbush, B., 3rd Functional interaction of the K$\mathrm{Cl}$ cotransporter $(\mathrm{KCC} 1)$ with the Na-K-Cl cotransporter in HEK-293 cells. American journal of physiology 1999, 276 (2), C328-C336.

(30) Mateus, A.; Matsson, P.; Artursson, P. A high-throughput cellbased method to predict the unbound drug fraction in the brain. $J$. Med. Chem. 2014, 57 (7), 3005-10.

(31) Adachi, J.; Kumar, C.; Zhang, Y.; Mann, M. In-depth analysis of the adipocyte proteome by mass spectrometry and bioinformatics. Mol. Cell. Proteomics 2007, 6 (7), 1257-73.

(32) Ye, F.; Zhang, H.; Yang, Y. X.; Hu, H. D.; Sze, S. K.; Meng, W.; Qian, J.; Ren, H.; Yang, B. L.; Luo, M. Y.; Wu, X.; Zhu, W.; Cai, W. J.; Tong, J. B. Comparative proteome analysis of 3T3-L1 adipocyte differentiation using iTRAQ-coupled 2D LC-MS/MS. J. Cell. Biochem. 2011, 112 (10), 3002-14.

(33) Prior, M. J.; Larance, M.; Lawrence, R. T.; Soul, J.; Humphrey, S.; Burchfield, J.; Kistler, C.; Davey, J. R.; La-Borde, P. J.; Buckley, M.; Kanazawa, H.; Parton, R. G.; Guilhaus, M.; James, D. E. Quantitative proteomic analysis of the adipocyte plasma membrane. J. Proteome Res. 2011, 10 (11), 4970-82.

(34) Freiwald, A.; Weidner, C.; Witzke, A.; Huang, S. Y.; Meierhofer, D.; Sauer, S. Comprehensive proteomic data sets for studying adipocyte-macrophage cell-cell communication. Proteomics 2013, 13 (23-24), 3424-8.

(35) Sawada, H.; Takami, K.; Asahi, S. A toxicogenomic approach to drug-induced phospholipidosis: analysis of its induction mechanism and establishment of a novel in vitro screening system. Toxicol. Sci. 2005, 83 (2), 282-292.

(36) Milo, R. What is the total number of protein molecules per cell volume? A call to rethink some published values. BioEssays 2013, 35 (12), 1050-5.

(37) Wisniewski, J. R.; Hein, M. Y.; Cox, J.; Mann, M. A "proteomic ruler" for protein copy number and concentration estimation without spike-in standards. Mol. Cell. Proteomics 2014, 13 (12), 3497-506.

(38) Logan, R.; Funk, R. S.; Axcell, E.; Krise, J. P. Drug-drug interactions involving lysosomes: mechanisms and potential clinical implications. Expert Opin. Drug Metab. Toxicol. 2012, 8 (8), 943-58.

(39) Yabe, Y.; Galetin, A.; Houston, J. B. Kinetic characterization of rat hepatic uptake of 16 actively transported drugs. Drug metabolism and disposition: the biological fate of chemicals 2011, 39 (10), 1808-14.

(40) Longhi, R.; Corbioli, S.; Fontana, S.; Vinco, F.; Braggio, S.; Helmdach, L.; Schiller, J.; Boriss, H. Brain tissue binding of drugs: evaluation and validation of solid supported porcine brain membrane vesicles (TRANSIL) as a novel high-throughput method. Drug metabolism and disposition: the biological fate of chemicals 2011, 39 (2), 312-21.

(41) Kalvass, J. C.; Maurer, T. S. Influence of nonspecific brain and plasma binding on CNS exposure: implications for rational drug discovery. Biopharm. Drug Dispos. 2002, 23 (8), 327-338.

(42) Law, V.; Knox, C.; Djoumbou, Y.; Jewison, T.; Guo, A. C.; Liu, Y.; Maciejewski, A.; Arndt, D.; Wilson, M.; Neveu, V.; et al. DrugBank 4.0: shedding new light on drug metabolism. Nucleic Acids Res. 2014, 42 (D1), D1091-D1097.

(43) Obach, R. S.; Lombardo, F.; Waters, N. J. Trend analysis of a database of intravenous pharmacokinetic parameters in humans for 670 drug compounds. Drug metabolism and disposition: the biological fate of chemicals 2008, 36 (7), 1385-405.

(44) Vance, J. E. Phospholipid synthesis and transport in mammalian cells. Traffic 2015, 16 (1), 1-18.
(45) Zhang, Y.; Baycin-Hizal, D.; Kumar, A.; Priola, J.; Bahri, M.; Heffner, K. M.; Wang, M.; Han, X.; Bowen, M. A.; Betenbaugh, M. J. High-Throughput Lipidomic and Transcriptomic Analysis To Compare SP2/0, CHO, and HEK-293 Mammalian Cell Lines. Anal. Chem. 2017, 89 (3), 1477-1485.

(46) Lagoutte, D.; Nicolas, V.; Poupon, E.; Fournet, A.; Hocquemiller, R.; Libong, D.; Chaminade, P.; Loiseau, P. M. Antifungal canthin-6-one series accumulate in lipid droplets and affect fatty acid metabolism in Saccharomyces cerevisiae. Biomed. Pharmacother. 2008, 62 (2), 99-103.

(47) Zhang, I.; Cui, Y.; Amiri, A.; Ding, Y.; Campbell, R. E.; Maysinger, D. Pharmacological inhibition of lipid droplet formation enhances the effectiveness of curcumin in glioblastoma. Eur. J. Pharm. Biopharm. 2016, 100, 66-76.

(48) Rak, S.; De Zan, T.; Stefulj, J.; Kosovic, M.; Gamulin, O.; Osmak, M. FTIR spectroscopy reveals lipid droplets in drug resistant laryngeal carcinoma cells through detection of increased ester vibrational bands intensity. Analyst 2014, 139 (13), 3407-15.

(49) Fujimoto, T.; Ohsaki, Y.; Cheng, J.; Suzuki, M.; Shinohara, Y. Lipid droplets: a classic organelle with new outfits. Histochem. Cell Biol. 2008, 130 (2), 263-79.

(50) Martin, S.; Parton, R. G. Lipid droplets: a unified view of a dynamic organelle. Nat. Rev. Mol. Cell Biol. 2006, 7 (5), 373-8.

(51) Velkov, T.; Lim, M. L.; Horne, J.; Simpson, J. S.; Porter, C. J.; Scanlon, M. J. Characterization of lipophilic drug binding to rat intestinal fatty acid binding protein. Mol. Cell. Biochem. 2009, 326 (12), 87-95.

(52) Chuang, S.; Velkov, T.; Horne, J.; Porter, C. J.; Scanlon, M. J. Characterization of the drug binding specificity of rat liver fatty acid binding protein. J. Med. Chem. 2008, 51 (13), 3755-64.

(53) Velkov, T.; Horne, J.; Laguerre, A.; Jones, E.; Scanlon, M. J.; Porter, C. J. Examination of the role of intestinal fatty acid-binding protein in drug absorption using a parallel artificial membrane permeability assay. Chem. Biol. 2007, 14 (4), 453-65.

(54) Rowland, A.; Knights, K. M.; Mackenzie, P. I.; Miners, J. O. Characterization of the binding of drugs to human intestinal fatty acid binding protein (IFABP): potential role of IFABP as an alternative to albumin for in vitro-in vivo extrapolation of drug kinetic parameters. Drug metabolism and disposition: the biological fate of chemicals 2009, 37 (7), 1395-403.

(55) Gornicka, A.; Fettig, J.; Eguchi, A.; Berk, M. P.; Thapaliya, S.; Dixon, L. J.; Feldstein, A. E. Adipocyte hypertrophy is associated with lysosomal permeability both in vivo and in vitro: role in adipose tissue inflammation. American journal of physiology. Endocrinology and metabolism 2012, 303 (5), E597-606.

(56) Coury, F.; Zenger, S.; Stewart, A. K.; Stephens, S.; Neff, L.; Tsang, K.; Shull, G. E.; Alper, S. L.; Baron, R.; Aliprantis, A. O. SLC4A2-mediated $\mathrm{Cl}-/ \mathrm{HCO} 3-$ exchange activity is essential for calpain-dependent regulation of the actin cytoskeleton in osteoclasts. Proc. Natl. Acad. Sci. U. S. A. 2013, 110 (6), 2163-8. 